\title{
Improving Dental Caries Detection by Optimizing Source-Detector Localization Using Laser Diffuse Reflectance
}

\author{
Ahmed L. Abdel Gawad*, Ashraf F. El-Sherif ${ }^{\dagger}$, Yasser El-Sharkawy ${ }^{\ddagger}$, H.S. Ayoub ${ }^{\S}$, \\ and Mahmoud F. Hassan ${ }^{* *}$
}

\begin{abstract}
In this work, a laser scattering method was used for measuring the optical properties of dental caries using $\mathrm{He}-\mathrm{Ne}$ laser. A spectrometer setup was built to test the optical characteristics of the molar tooth decay. The measurements yield the reflectance, absorbance and the transmittance of sound and decayed tooth. Monte-Carlo simulation was used to investigate and provide further analysis of the resultant measurements of the spectrometer. The results differentiate between sound and caries and enable to predict the exact localization of source-detector pair relative to the examined tooth, in order to help in performing optical imaging for in-vivo early detection of caries.
\end{abstract}

Keywords: Human Caries detection, diffuse reflectance, Laser Spectrophotometry, MonteCarlo simulation, Optical Characterization.

\section{Introduction}

In spite of the diversity of techniques used for the diagnosis of dental caries[1-5], limited number of methods has proven partial success in clinical practice[6]. Among most promising technique, one can mention simple optical trans-illumination of the suspected tooth that significantly helps to differentiate between mild and Sound enamel. Nevertheless, this technique encounters many problems, especially when attempt to automate the diagnosis, that mostly depends on human skills and expertise of the examiner. To enable the design and automation of a reliable caries detection technique based on laser scattering method, it was a must to characterize the optical properties of sound and pre-caries dental map of the examined tooth primarily through in-vitro measurements. In the present work, we decided to apply laser spectrophotometry to investigate the optical reflectance, absorbance and transmittance of the tooth using a low power He-Ne laser beam. The reason behind the use of such laser is the fact that red radiation of $635 \mathrm{~nm}$ is probably the safest visual color on oral cavity[7]. At the end of the procedure, it will be possible to apply Mont-Carlo modeling to conclude the localization of the detection system relative to the tooth morphology.

\footnotetext{
Egyptian Armed Forces, Egypt; louayhassan1972@gmail.com

Egyptian Armed Forces, Egypt.

Egyptian Armed Forces, Egypt.

Department of Physics, Faculty of Science, Cairo University, Egypt.

Egyptian Armed Forces, Egypt.
} 


\section{Motivation}

Laser radiation can undergo a number of processes while propagating in dental tissues, including reflection, absorption, scattering and fluorescence[8]. These processes are the basic conceptional methodology for human caries detection in modern in-vivo examination trends. The common issue between different optical diagnosis methods, is that the optical caries detection system. This system usually consists of two main devices namely the illumination source and the optical detector. To The best of our knowledge, the relative position of these devices with respect to the examined teeth is the major factor on which the success of the used method depends on. Many researchers attempt to enhance the contrast between sound and caries teeth by changing the wavelength of the illumination source, rather than finding the best inspection tool localization metrology (i.e. radial and angular distance from the examined tooth [9-11]. Hence most high contrast optical inspection technique deployed ionizing ultraviolet radiation or infrared radiation with harmful thermal effect, we decided to use red He-Ne laser that is believed to be much safer on oral cavity, easily deployed and cost effective, in detecting caries with a higher contrast than that reported in other works relying on optimized detection localization[9-11]It worth to mention that the use of He-Ne laser in studying the diffuse reflectance of human bulk dentin and enamel to differentiate caries was reported by Zijp et al,[12]. Similarly, Rong Li, et al [13], used diffuse reflectance for the same purpose using double beam spectrophotometry was investigated. In both research works, double integrating sphere technique is used with conjunction of Kublka-Monk formulation. In our work, we use adding-doubling method to calculate the optical properties absorption coefficient $\mu_{\mathrm{a}}$, scattering coefficient $\mu_{\mathrm{s}}$. Using the spectrophotometer equation which is given by:

$\mathrm{T}+\mathrm{A}+\mathrm{R}=1$

where, $A$ is the absorbance and $T$ is the transmittance of the sample respectively, $R$ is the reflectance of the sample.

\section{Materials and Methods}

\subsection{Experimental setup}

In order to measure the optical reflectance and transmittance of the tooth under examination, a setup was design as shown in figure 1. The system consisted of He-Ne Laser (Spectra-Physics Uniphase) of $5 \mathrm{mw}$ output power, irradiating a just extracted fresh molar tooth using a focusing lens with numerical aperture of $100 \mathrm{~mm}$, a precise $\mathrm{x}-\mathrm{y}-\mathrm{z}$ translation stage is holding the sample vertically, and a spectrometer detector with a fiber optic reflectance probe (USB HR4000CG, Ocean Optics, Inc), with spectral resolution of $0.1 \mathrm{~nm}$ at central wavelength, CCD device, and a computer with Spectra Suite acquisition software is installed at two locations relative to the sample where the first location is in front of the reflected laser portion, while the second location is behind the sample confronting the transmitted laser beam portion. The setup as shown in Figure 1 was able to measure and differentiate between the reflectance and transmittance of sound/caries samples at different areal positions on the superficial outer surface of the examined tooth.

\subsection{Test Samples}

Twelve molar teeth, freshly extracted for different orthodontic reasons with no dental restorations from patients of an age range from 20 to 50 years old, which are all either sound or infected by caries were collected from a nearby dental clinic, following extraction they were stored in $\mathrm{Na}-\mathrm{Cl}$ solution $(0.9 \%)$ at room temperature. The extracted teeth were clinically examined by only one experienced dentist and were classified according to the visual criteria of the International Caries Detection \& Assessment System (ICDAS) [14], where those with visible cavitation deeper than 1-2 mm were considered cavitated caries teeth. Before optical 
measurements, which usually that take place in a period less than 3 days from extraction, teeth were taken out of the saline solution, and washed in running water, and brushed using a soft tooth brush to clean the teeth out of food particles and blood residuals, and then they were well dried using a cotton towel.

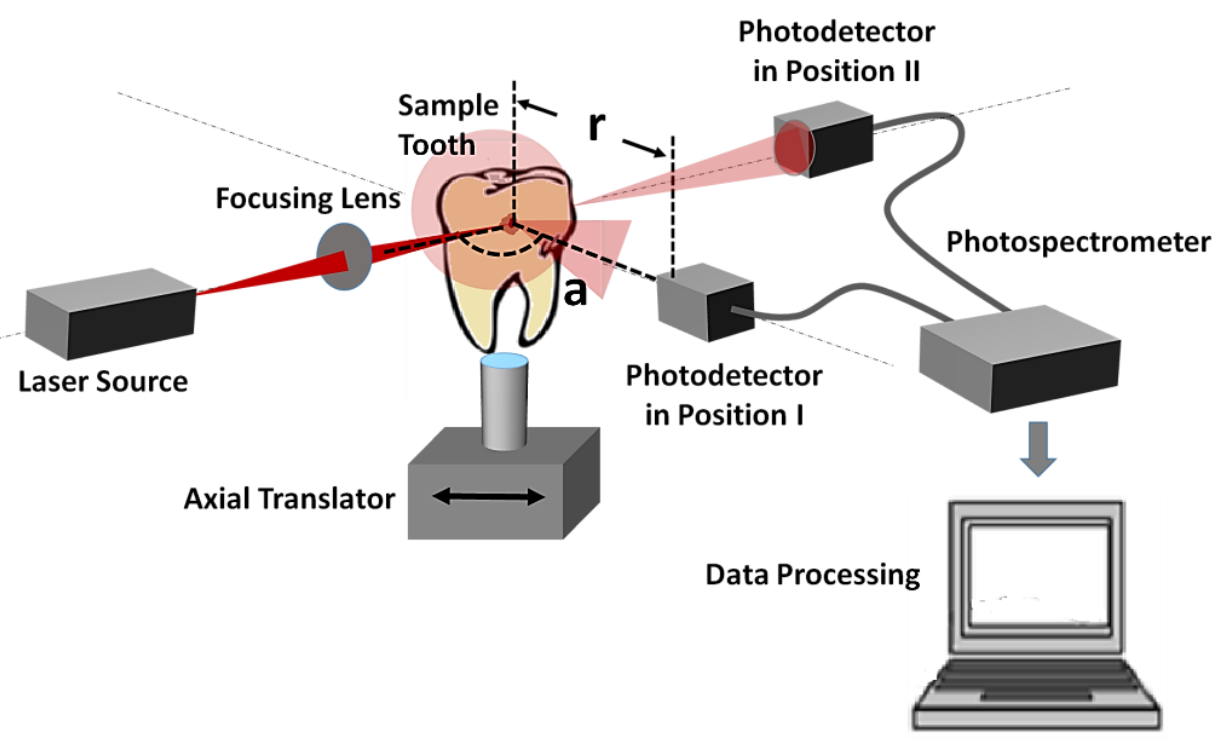

Fig. 1. The spectrometer test setup
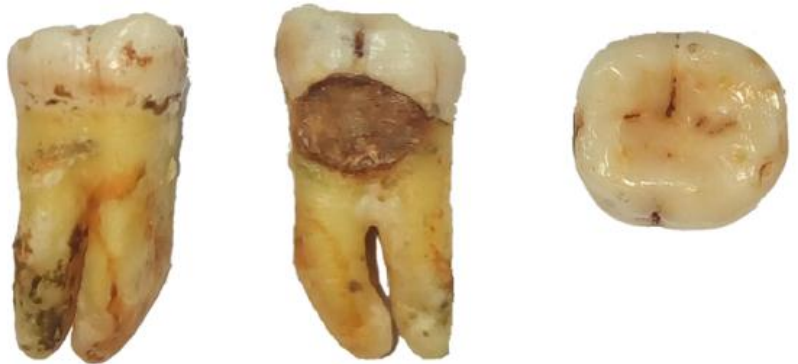

Fig. 2. Example of one of the lower molar tooth examined in this work

\subsection{Experimental Procedure}

At first, we performed an optical characterization for the used laser source, yielding a twonanometer spectral width as shown at figure 3.

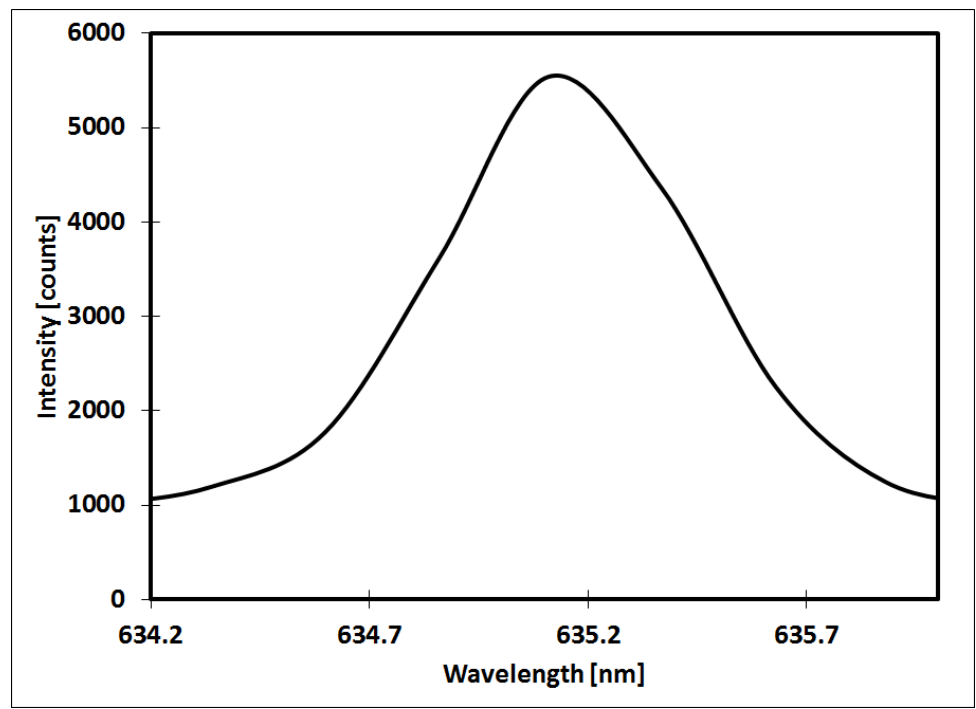

Fig. 3. Spectral bandwidth of the used laser source 
Then, a series of reflectance tests were carried on the 12 sound/caries tooth samples, where the detector was located at position I (as shown in fig.1) and angle $\mathrm{a}=$ zero. The tests investigated the reflectance topology of the sound/caries boundaries. The second series of tests investigated the transmittance and absorbance of the samples at the same analogy, where the detector was located at position II (as shown in Fig.1).

\section{Results and Discussion}

The measurements revealed the average transmittance as well as the average reflectance behavior of the whole twelve sound/caries samples, where the transmittance and the reflectance of the sound teeth were pretty much higher than those of the caries samples (as shown at Figs.4a and $4 b)$.

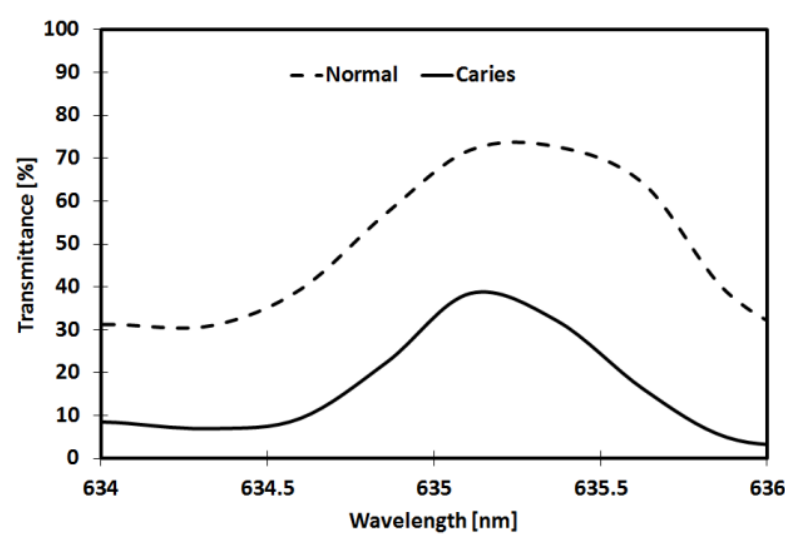

(a)

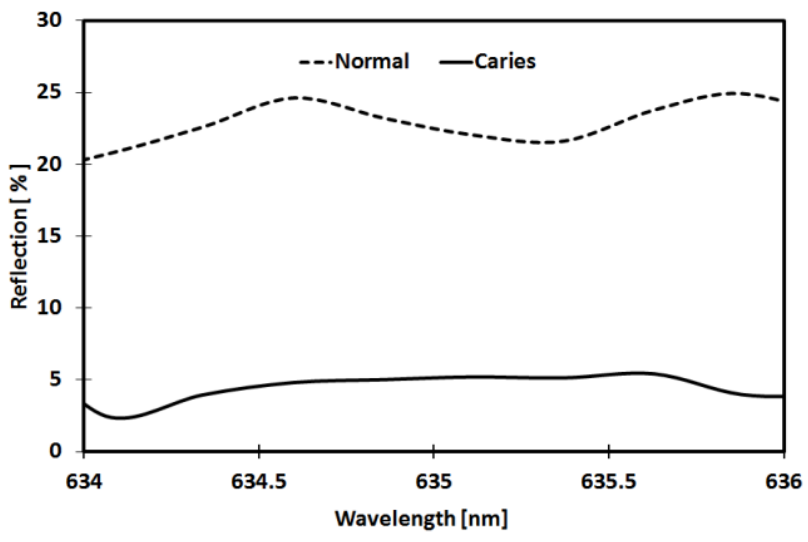

(b)

Fig. 4. (a) Average transmittance, (b) Average reflectance for the examined samples

These results could be explained on the bases of the opacity of the caries in the case of transmittance, where because most of the laser intensity was absorbed by the dark layers of the demineralized portion of the tooth (as shown at fig.5).

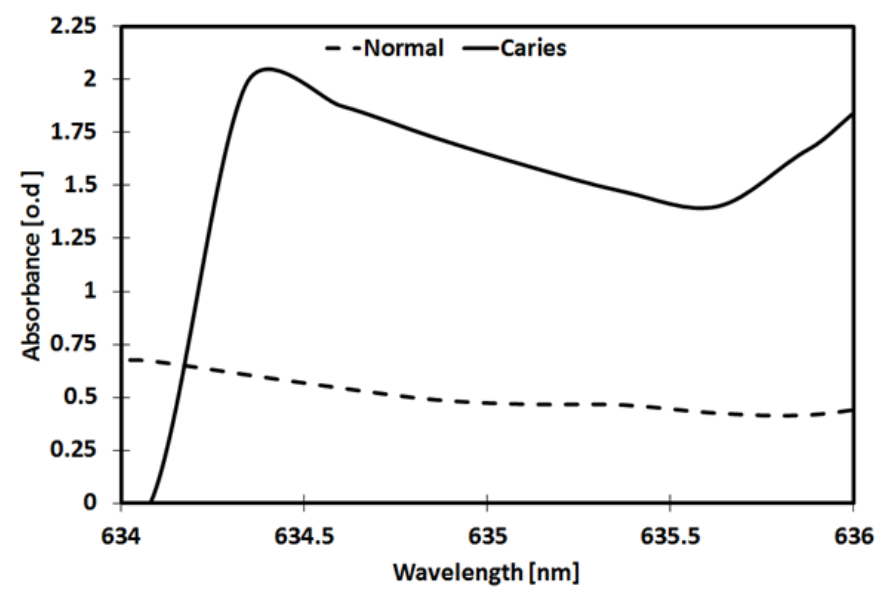

Fig. 5. Average absorbance of the examined samples

On the other hand, the concentrated presence of sound enamel and dentin at the examined tooth surface yielded much higher laser reflectance than that of the cavitated one. 


\subsection{Monte-Carlo analysis}

Stepping forward in our analysis, a Monte-Carlo model[15] was built in order to investigate the angular as well as the radial distance for source detector localization relative the tooth. A one layer Mont-Carlo model was built for sound teeth, using the appropriate grid elements and using the measured spectrophotometric average data for the sound teeth as model inputs as shown in Table 1

Table 1. Sound monolayer Monte-Carlo model inputs

\begin{tabular}{|c|c|c|c|c|c|c|c|}
\hline $\begin{array}{c}\text { Number } \\
\text { of } \\
\text { Layers }\end{array}$ & Tissue & $\begin{array}{c}\text { Scattering } \\
\text { Coefficient } \\
\boldsymbol{\mu}_{\mathrm{a}}\left(\mathbf{c m}^{-\mathbf{1}}\right)\end{array}$ & $\begin{array}{c}\text { Absorption } \\
\text { Coefficient } \\
\boldsymbol{\mu}_{\mathbf{s}}\left(\mathbf{c m}^{-\mathbf{1}}\right)\end{array}$ & $\mathbf{g}$ & $\mathbf{n}$ & $\begin{array}{c}\text { Thickness } \\
(\mathbf{c m})\end{array}$ & $\begin{array}{c}\text { Number } \\
\text { of } \\
\text { photons }\end{array}$ \\
\hline 1 & Sound & 0.468 & 22.06 & 0.97 & 1.62 & 1 & 1000000 \\
\hline
\end{tabular}

The same procedure was repeated for caries teeth, and another Monte-Carlo model is built, using the appropriate grid elements and using the measured spectrophotometric average data for the caries teeth as model inputs, as shown in Table 2

Table 2. Caries monolayer Monte-Carlo model inputs

\begin{tabular}{|c|c|c|c|c|c|c|c|}
\hline Layer & Tissue & $\begin{array}{c}\text { Scattering } \\
\text { Coefficient } \\
\boldsymbol{\mu}_{\mathrm{a}}\left(\mathbf{c m}^{-\mathbf{1}}\right)\end{array}$ & $\begin{array}{c}\text { Absorption } \\
\text { Coefficient } \\
\boldsymbol{\mu}_{\mathbf{s}}\left(\mathbf{c m}^{-\mathbf{1}}\right)\end{array}$ & $\mathbf{G}$ & $\mathbf{n}$ & $\begin{array}{c}\text { Thickness } \\
(\mathbf{c m})\end{array}$ & $\begin{array}{c}\text { Number } \\
\text { of } \\
\text { photons }\end{array}$ \\
\hline 1 & Caries & 1.592 & 5.2005 & 0.97 & 1.62 & 0.5 & 1000000 \\
\hline
\end{tabular}

The Monte-Carlo simulation produced the reflectance radial correlation (Fig. 6a) and angular correlation (Fig. 6b).

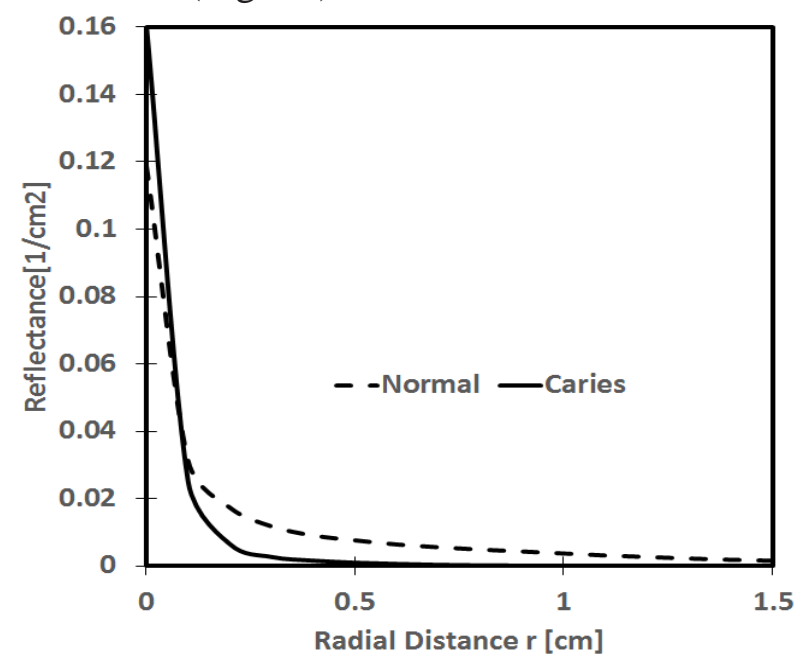

(a)

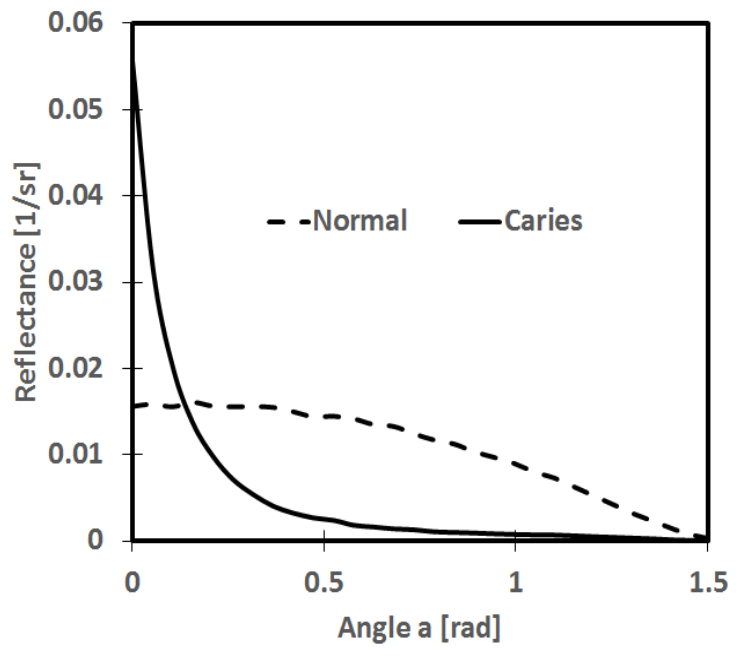

(b)

Fig. 6(a) Reflectance vs radial distance, (b) Reflectance vs angular distance

The simulation reveals that at a certain radial distance and a specific angle $(r=0.15 \mathrm{~cm}$, $\mathrm{a}=0.2 \mathrm{rad}$ ), the reflactance of the teeth shows an inflection behavior in which the reflectance of the caries appears to be higher than that of the sound just before the stated inflection point. At this point both reflactance are equal, but after this point the reflactance of the sound teeth ecxeed that of the caries as previously demonstrated experimentally $(\mathrm{r}=2 \mathrm{~cm}, \mathrm{a}=0.5 \mathrm{rad}) . \mathrm{In}$ order to test this model we adjusted our setup below this radial and angular limit, the results came in 
agreement with the simulation. To find the optimum inspection position on the tooth surface, we use the axial translator to laterly scan the cavitated tooth in a $0.1 \mathrm{~cm}$ step yielding eight positions as shown at Fig.7b, where position 3 and 6 represents the boundaries of the caries inside the tooth. Then, we performed reflactance intensity measurments at each nominated position.

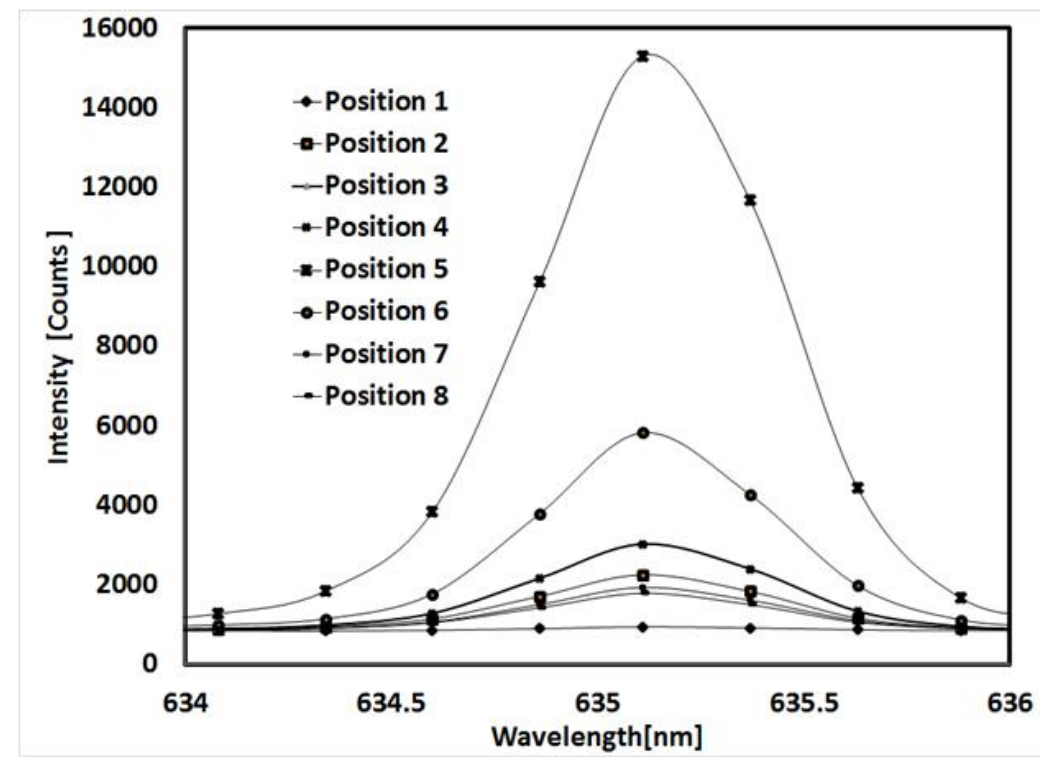

(a)

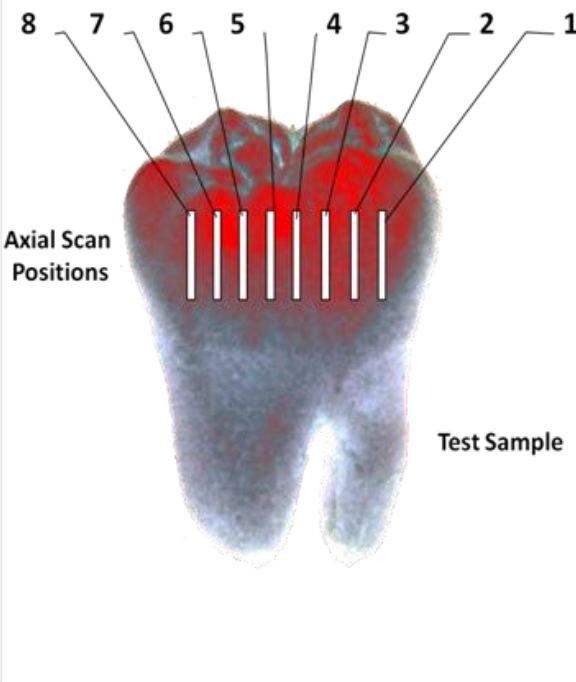

(b)

Fig.7 Optimization of laser source and detector localization, (a) Reflactance intensity for inspection positions, (b) Third molar selected inspection positions

The above experimental results (Fig.7a) shows that the optimum intensty contrast diffrentiating caries/sound tooth was given at positions 4 and 5 due to the increase of diffuse reflectance of the cavitated volume. This increase can be explaind in terms of the color contrast of sound enamel and the cavitated yellowish dentine.

\section{Conclusion}

Diffuse reflectance spectrometer technique employing He-Ne laser wavelength can be used to differentiate between sound and caries tooth if the position of source/detector is proparly localized. This was acheaved using Monte-Carlo simulation to treat spectrometer data. The simulation reveals critical angular and radial locus relative to the examined tooth. The results of the current work can be used to help in designing efficient, low cost in-vivo caries detection tools, based on safe laser wavelength in the visable range such as $\mathrm{He}-\mathrm{Ne}$, and using fiber optic waveguids to inspect tooth caries in the oral cavity.

\section{References}

[1] Price, J.B., A Review of Dental Caries Detection Technologies. 2013.

[2] El-Sharkawy, Y.H. and A.F. El Sherif, Photoacoustic diagnosis of human teeth using interferometric detection scheme. Optics \& Laser Technology, 2012. 44(5): p. 15011506.

[3] El-Sharkawy, Y.H. and A.F. El-Sherif. Human teeth model using photoacoustic frequency response. in SPIE BiOS. 2012. International Society for Optics and Photonics.

[4] El-Sherif, A.F., W.M.A. Aziz, and Y.H. El-Sharkawy. Multivariate image analysis of laser-induced photothermal imaging used for detection of caries tooth. in SPIE Optical Engineering + Applications. 2010. International Society for Optics and Photonics. 
[5] El-Sherif, A.F. and Y.H. El-Sharkawy. Laser-induced photothermal technique used for detection of caries in human tooth. in Biomedical Optics (BiOS) 2008. 2008. International Society for Optics and Photonics.

[6] Walsh, L., The current status of laser applications in dentistry. Australian dental journal, 2003. 48(3): p. 146-155.

[7] Parker, S., Laser regulation and safety in general dental practice. British dental journal, 2007. 202(9): p. 523-532.

[8] NIKONENKO, N., OPTICAL PROPERTIES OF SKIN, TOOTH TISSUES AND DENTAL MATERIALS.rep.bsmu.by/xmlui/bitstream/handle/BSMU/7985/366519-инбp..pdf?sequence

[9] Hibst, R. and K. Konig, Device for detecting dental caries. 1994, Google Patents.

[10] Bakhmutov, D., et al., Early dental caries detection by fluorescence spectroscopy. Laser Physics Letters, 2004. 1(11): p. 565.

[11] Zijp, J. and J. Ten Bosch, Angular dependence of HeNe-laser light scattering by bovine and human dentine. Archives of oral biology, 1991. 36(4): p. 283-289.

[12] Zijp, J., J. Ten Bosch, and R. Groenhuis, HeNe-laser light scattering by human dental enamel. Journal of dental research, 1995. 74(12): p. 1891-1898.

[13] Li, R., et al., Optical properties of enamel and translucent composites by diffuse reflectance measurements. Journal of dentistry, 2012. 40: p. e40-e47.

[14] Shivakumar, K., S. Prasad, and G. Chandu, International Caries Detection and Assessment System: A new paradigm in detection of dental caries. Journal of Conservative Dentistry, 2009. 12(1): p. 10.

[15] Wang, L. and S.L. Jacques, Monte Carlo modeling of light transport in multi-layered tissues in standard $C$. The University of Texas, MD Anderson Cancer Center, Houston, 1992. 